Article

\title{
Interaction of Hadronic Dark Matter with Nucleons and Leptons
}

\author{
Vitaly Beylin 1 and Vladimir Kuksa * \\ Research Institute of Physics, Southern Federal University, Pr. Stachky 194, 344090 Rostov-on-Don, Russia; \\ vitbeylin@gmail.com \\ * Correspondence: vkuksa47@mail.ru
}

Received: 17 February 2020; Accepted: 3 March 2020 ; Published: 5 April 2020

\begin{abstract}
We analyze the low-energy Lagrangian of hadronic dark matter interaction with nucleons and leptons. The analysis was fulfilled within the framework of the effective meson-exchange model, which is based on dynamic realization of $S U(3)$-symmetry. Using this Lagrangian, we calculate the cross-section of low-energy scattering of nucleons on hadronic dark matter particles. Effective vertex of $W$-boson interaction with new hadrons is constructed and the cross-section of lepton scattering on dark matter particles is calculated.
\end{abstract}

Keywords: dark matter; strong interaction; hadrons

\section{Introduction}

The problem of dark matter description inevitably leads to the Standard Model (SM) extension. Usually, the dark matter (DM) candidates are considered as stable or long-lived massive neutral particles interacting weakly with the ordinary ones (WIMPs). The last measurements of the cross-section of WIMP-nucleon scattering [1] bring strong experimental restrictions on the cross-section value, excluding some models of WIMPs. Some other scenarios are discussed in a papers, for example, the scenarios with strongly interacting new heavy particles [2-4]. Extensions of the SM with hadronic or hadron-like heavy particle as the dark matter (DM) carrier [5-8] are also suggested. In these scenarios, new particles consist of new heavy quark and the standard light quark. New quarks appear, for example, in the extension of the SM with 4-th generation [9-12], in the extension with singlet quark [5] and in the chiral-symmetric models [6] (see, also, the review [7] and references therein). Experimental and theoretical premises of new heavy hadron existence were discussed in [8]. The main properties and low-energy phenomenology of the hadronic DM were described in [5-7]. It was underlined there that repulsive asymptotic of DM particles interactions with nucleons prevents to form coupled states of these particles with ordinary cold matter. This effect makes it possible to escape contradictions with rigid cosmochemical restrictions on anomalous hydrogen and helium $[7,8]$.

In order to describe possible signals of the hadronic DM, we should develop the model of new hadrons interaction with usual matter. Here, we consider the interaction of the hadronic DM particles with nucleons and leptons. The Lagrangian of interaction is analyzed and represented in detail in the framework of the effective low-energy meson-exchange approach, which is based on dynamic realization of $S U(3)$-symmetry. In this consideration, we take into account that heavy DM is cold and essentially non-relativistic now, therefore the effective meson-exchange model can be applied for the description of the DM-nucleon strong interaction. The validity of this approach is restricted by the value of momentum transfer, $q^{2} \leq m^{2}$, where $m$ is the mass of light carrier of interaction in the intermediate state, $m \sim 1 \mathrm{GeV}$. Kinematical analysis of the nucleon scattering on a cold DM revealed that this condition is realised in the local reference frame which is comooving with non-relativistc galactic matter. Using the model Lagrangian of DM interaction with nucleons and leptons, we derived 
analytical expressions for the cross-section of their scattering on the DM particles. These cross-sections will be used in the future analysis and description of the DM interactions with usual matter.

The paper is organised as follows. In Section 2, we represent effective Lagrangian of baryon-meson and DM-meson interactions at low energy. The analytical expressions for the cross-sections of nucleons scattering off new heavy hadrons are derived in Section 3. The Lagrangian of the DM interaction with the gauge boson $W$ is constructed in Section 4, where the cross-section of the DM-electon scattering is calculated. Some conclusions are represented in Section 5.

\section{Meson-Exchange Model of New Hadrons Interaction with Nucleons}

At hadronization stage of evolution, new heavy quarks, $Q$, form coupled states with ordinary light quarks, $q$. Full classification of these heavy states was considered in Ref. [7], where quark composition of two-quark (meson) and three-quark (fermion) states was represented for the case of up- and down-types of new quark $Q$. In analogy with the standard heavy-light mesons, the states of type $(q Q)$ can be scalar, pseudoscalar and vector new heavy mesons. They possess effective vertexes of interaction with gauge vector bosons and with various hadron states (standard and new). The first type of interactions is defined quantitatively by electro-weak coupling constant and dominates in high energy processes at short distances, $R_{W} \sim 1 / M_{W}$. The second type of interactions is described by strong couplings and dominates in low-energy processes at long distances $R_{S} \sim 1 / m_{n}$, where $m_{n}$ is the mass of nucleon. Here, we consider in detail the second type of low-energy interactions in the framework of effective low-energy model, which can be applied to describe interaction of cold DM with nucleons.

It is known that low-energy nucleons interaction can be described in the framework of effective Yukawa's type theory. The interaction between nucleons in this theory is explained by $\pi$-meson exchange. This approach was developed by including singlet vector $(\omega)$ and scalar $\left(f_{0}\right)$ mesons in Ref. [13]. An account of $\rho$-meson contribution was realized in the framework of $S U(2)$-gauge scheme in Ref. [14]. To consider the meson-exchange interaction of new hadrons with nucleons, we need vertexes which describe interaction of nucleons and new hadrons with mesons in the intermediate states. The first type of vertexes is represented in [15], where their description was developed on the base of the gauge scheme $U(1) \times S U(3)$. There, $U(1)$ is the group of semistrong interaction corresponding to the exchange by singlet vector meson, and $S U(3)$ is group of baryon unitary symmetry which includes exhange by octet of vector mesons. It was demonstrated in [15] that the baryon-meson model of interactions successfully describes the low-energy hadrons phenomenology. Namely, the model gives three reasonable mass relations in meson and baryon sectors, and describes the decays of vector mesons in good agreement with experimental data. This scheme also contains principal properties of nucleon-nucleon interactions and makes it possible to introduce and operate with the minimal set of model parameters. Here, we apply this approach to the low-energy description of nucleon scattering on the DM particles. First of all, we represent the main elements of the gauge model of baryon-meson interactions. The particles content of the model is the following:

1. Octet of baryons $B=\left(N, \Theta, \Sigma, \Lambda^{0}\right)$ (adjoint representation of strong group $\left.S U(3)\right)$.

2. Singlet $V^{0}$ and octet $V=\left(\rho, \phi^{0}, K^{*}, \bar{K}^{*}\right)$ of vector mesons (gauge fields).

3. Three triplets of scalar mesons $H=\left(a_{0}, f_{0}, f_{0}^{\prime}, K_{0}, \bar{K}_{0}\right)$ (higgs fields).

4. Octet of pseudoscalar fields $\Phi=(\pi, \eta, K, \bar{K})$ (adjoint representation).

The full baryon-meson Lagrangian is rather cumbersome, and here we represent only its main parts which will be used in further considerations. The interactions of baryon with vector mesons are described in accordance with the gauge scheme:

$$
L_{B V}=i \bar{B} \gamma^{\mu}\left\{\partial_{\mu} B-i \kappa V_{\mu}^{0}-\frac{i}{\sqrt{2}} g\left[V_{\mu}, B\right]\right\}
$$


In (1), the matrices $B$ and $V$ represent octets of baryons $B=\lambda_{i} B^{i} / \sqrt{2}$ and vector mesons $V_{\mu}=$ $\lambda_{i} V_{\mu}^{i} / \sqrt{2}$, where $\lambda_{i}$ are Hell-Mann matrixes, $i=1,2, \ldots 8$, and $\left[V_{\mu}, B\right]$ is commutator of matrices $V_{\mu}$ and $B$. As a result of unitary symmetry breaking, octet of baryons $B$ is splitted to the doublet of nucleon $N=(p, n)$, doublets of $\Theta$-particles $\Theta=\left(\Theta^{0}, \Theta^{-}\right)$, triplet $\Sigma=\left(\Sigma^{+}, \Sigma^{0}, \Sigma^{-}\right)$and neutral singlet $\Lambda^{0}$. Analogously, octet of vector mesons $V$ is splited to the doublets $K^{*}=\left(K^{*+}, K^{* 0}\right)$ and its antiparticles $\bar{K}^{*}=\left(\bar{K}^{*-}, \bar{K}^{* 0}\right)$, triplet $\rho=\left(\rho^{+}, \rho^{0}, \rho^{-}\right)$and singlet $\phi^{0}$. After the unitary symmetry breaking the initial singlet fields $V^{0}$ and $\phi^{0}$ are mixed and form two physical vector states $\omega(782)$ and $\phi(1020)$. It is known that the last meson consists of mainly s-quarks, $\phi(1020)=(s \bar{s})$ and the coupling of $\phi(1020)$ with nucleons is zero. From this condition, for gauge couplings it follows the relation:

$$
\kappa=\frac{\sqrt{3}}{2} g \tan \theta,
$$

where $\theta$ is the angle of $V^{0}-\phi^{0}$ mixing. The part of physical Lagrangian which describes the interaction of nucleons with vector mesons is as follows:

$$
\begin{aligned}
L_{N V} & =g_{\omega} \omega_{\mu}\left(\bar{p} \gamma^{\mu} p+\bar{n} \gamma^{\mu} n\right)+\frac{1}{2} g \rho_{\mu}^{0}\left(\bar{p} \gamma^{\mu} p-\bar{n} \gamma^{\mu} n\right) \\
& +\frac{1}{\sqrt{2}} g \rho_{\mu}^{+} \bar{p} \gamma^{\mu} n+\frac{1}{\sqrt{2}} g \rho_{\mu}^{-} \bar{n} \gamma^{\mu} p,
\end{aligned}
$$

where $g_{\omega N}=\sqrt{3} g / 2 \sin \theta$. The fitting of model parameters from decays of vector mesons and the $\omega-\phi$ mixing results in the following values: $g^{2} / 4 \pi \approx 3.4$ and $\sin \theta \approx 0.63$.

The Lagrangian of interaction of baryon octet $B$ with pseudoscalar octet $\Phi=\lambda_{i} \Phi^{i} / \sqrt{2}$ and singlet $\varphi^{0}$ has the form:

$$
L_{B \Phi}=f_{0} \varphi^{0}\left(\bar{B} \gamma^{5} B\right)+f_{8}\left(\bar{B} \gamma^{5} B \Phi\right)+d_{8}\left(\bar{B} \gamma^{5} \Phi B\right) .
$$

The part of physical Lagrangian which follows from (4) and describes the interaction of nucleons with $\pi$-mesons is as follows:

$$
L_{N \pi}=i g_{\pi} \pi^{0}\left(\bar{p} \gamma^{5} p-\bar{n} \gamma^{5} n\right)+\frac{i}{\sqrt{2}} g_{\pi}\left(\pi^{+} \bar{p} \gamma^{5} n+\pi^{-} \bar{n} \gamma^{5} p\right),
$$

where the value of coupling constant $g^{2} / 4 \pi \approx 14$ is fitted from $\pi N$-scattering cross-section at the low energy.

Three fundamental representations of complex scalar fields are combined into $(3 \times 3)$-matrix $H$. The part of initial Lagrangian describing interactions of baryons and scalar mesons has the form:

$$
L_{B H}=a_{1}\left(H^{+} \bar{B} B H\right)+a_{2}\left(\bar{B} H H^{+} B\right) .
$$

After the breaking of $S U(3)$-symmetry $H=\Phi+v$, where $v$ is diagonal matrix of parameters of shifting, $v=\operatorname{diag}\left(v_{1}, v_{2}, v_{3}\right), v_{1}=v_{2}$. Nine degrees of freedom from $H$ convert to longitudinal polarizations of vector fields providing their masses. The remaining nine degrees of freedom form the nonet of physical states, i.e., scalar mesons $\Phi=\left(a_{0}, f_{0}, f_{0}^{\prime}, K^{0}, \bar{K}^{0}\right)$. Their interactions with nucleons are described by the following Lagrangian:

$$
L_{N \Phi}=g_{f} f(\bar{p} p+\bar{n} n)+g_{a} a(\bar{p} p-\bar{n} n)+\frac{g_{a}}{\sqrt{2}}\left(a^{+} \bar{p} n+a^{-} \bar{n} p\right)
$$

where $f=f_{0}(980), a=a_{0}(980)$, and interaction coupling $g_{f} \sim g_{a} \sim 1$.

The interaction of new hadrons with ordinary mesons can be considered using the same $S U(3)$-symmetry. To incorporate interactions of new hadrons with vector mesons, we apply the gauge scheme of dynamical realization of this symmetry. Then, the doublet of new heavy mesons $M_{(2)}=\left(M^{0}, M^{-}\right)$should be extended to triplet as the fundamental representation of $S U(3)$ group. 
This extension can be fulfilled by including standard s-quark into composition of new heavy mesons: $M_{(3)}=\left(M^{0}, M^{-}, M_{s}^{-}\right)$, where $M^{0}=(\bar{U} u), M^{-}=(\bar{U} d)$ and $M_{s}^{-}=(\bar{U} s)$. Here, we should note that $S U(2)$ isotopic symmetry of nucleons, which consist of $u$ - and $d$-quarks, is exact with respect to strong interaction. The extension of the isotopic symmetry to unitary $S U(3)$ group has an approximate character. It, however, allows to analyze the symmetry of baryon octet consisting of $u, d$ and $s$ quarks. Significant violation of $S U(3)$-symmetry is caused by large value of mass-splitting of the first and second quark generation. In our case, the heavy hadrons $M=(q U)$ consist of light $(u, d, s)$ and heavy $(U)$ quarks, so the mass-splitting is negligible. Thus, the symmetry $S U(3)$ is near exact and we can consider its dynamical (gauge) realization. At low energy, the intermediate meson states can be constructed by the standard light quarks.

Lagrangian of low-energy interactions of $M$-particles with vector mesons $V$ is defined in the standard gauge form:

$$
L_{M_{3} V}=\left(D^{\mu} M_{3}\right)^{\dagger} D_{\mu} M_{3}, \quad D_{\mu} M_{3}=\left(\partial_{\mu}-i t V_{\mu}^{0}-\frac{i g}{\sqrt{2}} V_{\mu}\right) M_{3},
$$

where $(A)^{\dagger}$ is Hermitian conjugation and $t$ is new gauge parameter. From (8), it follows the physical Lagrangian of interaction of $M$-particles with vector mesons:

$$
\begin{aligned}
L_{M V} & =i g_{\omega M} \omega^{\mu}\left(\bar{M}^{0} M_{, \mu}^{0}-\bar{M}_{, \mu}^{0} M^{0}+M_{, \mu}^{+} M^{-}-M^{+} M_{, \mu}^{-}\right) \\
& +\left(i g_{\omega M_{s}} \omega_{\mu}+i g_{\phi M_{s}} \phi_{\mu}\right)\left(M_{s}^{+} M^{-, \mu}-M^{+, \mu} M_{s}^{-}\right) \\
& +\left(\frac{i g}{2} \rho_{\mu}^{0}+i g_{\phi M} \phi_{\mu}\right)\left(\bar{M}^{0} M_{, \mu}^{0}-\bar{M}_{, \mu}^{0} M^{0}+M_{, \mu}^{+} M^{-}-M^{+} M_{, \mu}^{-}\right) \\
& +\frac{i g}{\sqrt{2}} \rho^{+\mu}\left(\bar{M}^{0} M_{, \mu}^{-}-\bar{M}_{, \mu}^{0} M^{-}\right)+\frac{i g}{\sqrt{2}} \rho^{-\mu}\left(M^{+} M_{, \mu}^{0}-M_{, \mu}^{+} M^{0}\right) .
\end{aligned}
$$

To define coupling constant of physical fields we should take into account quark composition of vector meson $\phi=(s \bar{s})$ and new mesons $M^{0}=(\bar{U} u), M^{-}=(\bar{U} d)$. Then we get $g_{\phi M}=0$ and following relations:

$$
g_{\phi M}=t \sin \theta-\frac{g}{2 \sqrt{3}} \cos \theta=0 \Longrightarrow t=\frac{g}{2 \sqrt{3}} \cot \theta=\frac{\kappa}{3} .
$$

An account of quark composition of new meson $M_{s}^{-}=(\bar{U} s)$ leads to the condition $g_{\omega M_{s}}=0$, that is:

$$
g_{\omega M_{s}}=\frac{g}{2 \sqrt{3} \sin \theta}\left(1-3 \sin ^{2} \theta\right)=0 \Longrightarrow \sin \theta=\frac{1}{\sqrt{3}} .
$$

Using Equations (10) and (11), we represent the rest constants coupling in the form:

$$
g_{\omega M}=\frac{g}{2 \sqrt{3} \sin \theta}=\frac{g_{\omega N}}{3}, g_{\phi M_{s}}=-\frac{\sqrt{3}}{2} g \cos \theta .
$$

Thus, the extension of low-energy baryon-meson model of interaction with triplet of new hadrons gives an additional information on coupling constants, namely, the relations (10)-(12). Here, we should note that the condition following from the right side of Equation (11) fixes the mixing angle value, $\sin \theta=1 / \sqrt{3} \approx 0.58$. This value, however, was fixed already in the low-energy part of the model [15], $\sin \theta \approx 0.63$, that is one can see an approximate self-consistency of the full model. Small discrepancy is caused by limited accuracy of baryon $S U(3)$-symmetry and low-energy approach with including of strange hadrons. It should be noted, olso, that the symmetry of heavy triplet $\left(M^{0}, M^{-}, M_{S}\right)$ is nearly exact due to super-heavy new quark $Q$, and we should accept the value of mixing angle, $\sin \theta=1 / \sqrt{3}$, as the more appropriate. 
Tri-linear interactions of new scalar (or pseudoscalar) particle with standard pseudoscalar mesons are absent because the vertexes of type $\Phi M M$ are forbidden by parity conservation. This is principal feature which differs new hadrons from nucleon case, where the vertex $\Phi N N$ is permitted (see expression (3)). So, the interaction of $M$-particle with nucleon through one-pion exchange is absent at the tree level.

Interactions of $M$-particles with scalar mesons in analogy with (6) are described by four-field Lagrangian:

$$
L_{M H}=h_{1}\left(M^{\dagger} M\right)\left(H^{\dagger} H\right)+h_{2}\left(M^{\dagger} H H^{\dagger} M\right) .
$$

As a result of shift $H=\Phi+\hat{v}$, where $\hat{v}=\operatorname{diag}\left(v_{1}, v_{2}, v_{3}\right)$, in Equation (13) appear the terms which describe the interactions of $M$-particles with the ordinary scalar mesons, for instance singlet $f_{0}$ and triplet $a_{0}$ :

$$
L_{M S}=g_{f M} f_{0}\left(\bar{M}^{0} M^{0}+M^{+} M^{-}\right)+g_{a M}\left(M^{\dagger} a_{0}^{k} \tau_{k} M\right), \quad k=1,2,3,
$$

where the value of coupling constants $g_{f M}$ and $g_{a M}$ are proportional to the value of shift $v \approx v_{a}$ in multiplet $H$ :

$$
g_{f M}=k_{f} v, g_{a M}=k_{a} v, k_{f, a} \sim 1, v \approx 0.1 m_{N} .
$$

As it will be shown further, this factor leads to significant suppression of $N M$-interaction through scalar meson exchange.

\section{Cross-section of Nucleons Scattering on New Hadrons}

The scattering of nucleons on new heavy hadrons can be analyzed at the level of one-meson-exchange diagrams with vector and scalar mesons in the intermediate t-channel states. To refresh, a diagram with intermediate pseudoscalar (pion) meson does not contribute at the tree level to prevent parity violation. So, this interaction is realized through two-pion exchange because the vertex of type $M M \Phi \Phi$ is permitted. However, the vertex of type $N N \Phi \Phi$ is absent in the model [15] and the two-pion exchange is possible at the loop level only. First of all, we consider elastic scattering of type $M N \rightarrow M N$, where $M=\left(M^{0}, M^{-}\right)$and $N=(p, n)$. If particles are non-relativistic, value of momentum transfer $Q^{2}=-q^{2}$ is defined by the expression:

$$
Q^{2} \approx k_{N}^{2}+p_{N}^{2}-2 \cos \beta k_{N} p_{N}
$$

where $p_{N}$ and $k_{N}$ are three-momenta of nucleon in initial and final states, and $\cos \beta$ is the angle of scattering. The value $Q^{2}$ can be expressed, also, in terms of heavy hadron momenta $p_{M}$ and $k_{M}$ by the same formula. In the center-of-mass system (CMS) $\vec{p}_{N}+\vec{p}_{M}=0$ and for the case of non-relativistic process we get the relation $v_{M} m_{M}=v_{N} m_{N}$, where $v_{M}+v_{N}=v_{r}$ is relative velocity. In the Galaxy rest system (the comoving system of frame) the DM particle velocity $v \sim 10^{-3}$ (in units of light velocity $c$ ), so for the case under consideration $v_{r} \sim 10^{-3}$. As a result, we have:

$$
v_{N}=v_{r} /\left(1+\frac{m_{N}}{m_{M}}\right) \approx v ; v_{M}=v_{r} /\left(1+\frac{m_{M}}{m_{N}}\right) \approx v \frac{m_{N}}{m_{M}} .
$$

So, in CMS $p_{N} \approx p_{M} \approx m_{N} v_{r}$ and from the energy conservation it follows $k_{N} \approx k_{M} \approx m_{N} v_{r}$. According to (16) maximal value of momentum transfer is $Q_{\max }^{2}=(p+k)^{2} \approx 4 m_{N}^{2} v_{r}^{2}$, that is $Q_{\max } \approx$ $m_{N} v_{r} \sim 10^{-3} m_{N}$. From this evaluation, it follows that momentum transfer much less the mass of intermediate mesons and the meson-exchange approach is relevant.

Now, we consider the process of proton elastic scattering on the DM particle, $p M^{0} \rightarrow p M^{0}$, which is defined by the $t$-channel diagrams with vector $V=\left(\omega, \rho^{0}\right)$ and scalar $S=\left(f_{0}, a_{0}^{0}\right)$ intermediate mesons. We check that the relation of scalar and vector contribution to the amplitude square is as follows:

$$
\frac{A_{S}^{2}}{A_{V}^{2}} \sim \frac{g_{s p}^{2} g_{s M}^{2}}{m_{M}^{2}} ; g_{s p}=k v_{1} \sim 0.1 m_{N}
$$


where $g_{s p}$ was defined in (12) and $g_{s M}$ is an unknown dimensionless coupling constant. For the case of realistic value $g_{s M} \sim 1$ the relation of contributions is

$$
\frac{A_{S}^{2}}{A_{V}^{2}} \sim 10^{-2} \frac{m_{N}^{2}}{m_{M}^{2}} \sim 10^{-10}
$$

The value of interference term is an order of $10^{-5}$, so we get strong suppression of the scalar contribution. Using the Lagrangian from the previous section, we calculate cross-section for the process $p M^{0} \rightarrow p M^{0}:$

$$
\sigma\left(p M^{0} \rightarrow p M^{0}\right)=\frac{g^{4} m_{p}^{2}}{16 \pi m_{V}^{4}}\left(1+\frac{1}{\sin ^{2} \theta}\right),
$$

where $V=\left(\omega, \rho^{0}\right), m_{V} \approx 0.75 \mathrm{GeV}, g^{2} / 4 \pi \approx 3.4$ and $\sin \theta=1 / \sqrt{3}$. As a result, we get rather large value, $\sigma\left(p M^{0} \rightarrow p M^{0}\right) \approx 0.9$ barn. However, the value of kinetic cross-section $(\sigma v)$ is suppressed by the factor $v_{r} \sim 10^{-3}$. Elastic cross-sections for other pairs of particles $p M^{-}, n M^{0}$ and $n M^{-}$are the same, because of coupling constants are the same, $m_{p} \approx m_{n}$ and $m\left(M^{0}\right) \approx m\left(M^{-}\right)$. Note, the cross-section of non-relativistic scattering does not depend on the mass $m_{M}$ and the angle of scattering. The processes of elastic skattering can be detected as nucleon recoil in the instruments with solid detector.

Further, we consider inelastic scattering of type $N_{1} M_{1} \rightarrow N_{2} M_{2}$, where $N_{i}=p, n$ and $M_{i}=$ $M^{0}, M^{-}$. These processes possess threshold when the sum of final masses exceed sum of initial masses. Because the mass splitting in both doublets, nucleons and new hadrons, is very small the kinematics of these processes is practically the same as in the elastic scattering. In particular, the value of momentum transfer is defined by Equation (16) and $Q_{\max } \approx m_{N} v_{r} \sim 10^{-3} m_{N}$. Let us consider the process $p M^{0} \rightarrow n M^{+}$where $M^{+}=\left(M^{-}\right)^{\dagger}=\bar{M}^{-}$. In this case, dominant contribution is defined by $t$-channel diagram with intermediate $\rho^{+}$and the cross-section can be represented in a form indicating the threshold existence explicitly:

$$
\sigma\left(p M^{0} \rightarrow n M^{+}\right)=\frac{g^{4} m_{n}}{8 \pi v_{r} m_{\rho}^{4}} \sqrt{2 m_{p}}\left[E_{p}-\delta m_{M}-\delta m_{N}\right]^{1 / 2},
$$

where $E_{p} \approx m_{p} v_{r}^{2} / 2, \delta m_{M}=m^{+}-m^{0}$ and $\delta m_{N}=m_{n}-m_{p} \approx 1.4 \mathrm{MeV}$. From the threshold kinetic energy $E_{p}^{t h r}=\delta m_{M}+\delta m_{N} \equiv \Delta m$ it follows the value of velocity at the threshold $v_{r}^{t h r}=\sqrt{2 \Delta m / m_{p}}$. For the case $\Delta m=10 \mathrm{MeV}$ we get rather large relative velocity $v_{r}^{\text {thr }}=0.1$, which is much larger than the DM particles velocity at the moment, $v_{r} \sim 10^{-3}$. So, the process can not be registered by charged particles detectors. The expression (21) can be rewritten in more simple form:

$$
\sigma\left(p M^{0} \rightarrow n M^{+}\right)=\frac{g^{4} m_{N}^{2}}{8 \pi m_{\rho}^{4}}\left[1-\frac{\delta m_{M}+\delta m_{N}}{E_{p}}\right]^{1 / 2} .
$$

The full process of proton scattering on $M^{0}$ looks as follows: $p M^{0} \rightarrow n M^{+} \rightarrow e^{-} p e^{+} M^{0}$, that is an obvious signal of the process is electron-positron pair production in the final state.

Expression for cross-section of inverse process, $n M^{+} \rightarrow p M^{0}$, can be directly derived from the Equation (22) by the change $\Delta m \rightarrow-\Delta m$ :

$$
\sigma\left(n M^{+} \rightarrow p M^{0}\right)=\frac{g^{4} m_{N}^{2}}{8 \pi m_{\rho}^{4}}\left[1+\frac{\delta m_{M}+\delta m_{N}}{E_{p}}\right]^{1 / 2} .
$$

This is non-threshold reaction with unstable (metastable) particles in the initial state. The expression for cross-section of the process $n M^{0} \rightarrow p M^{-}$follows from (22) with the change $\delta m_{N} \rightarrow-\delta m_{N}:$

$$
\sigma\left(n M^{0} \rightarrow p M^{-}\right)=\frac{g^{4} m_{N}^{2}}{8 \pi m_{\rho}^{4}}\left[1-\frac{\delta m_{M}-\delta m_{N}}{E_{p}}\right]^{1 / 2} .
$$


In this reaction, the threshold energy is $E_{p}^{t h r}=\delta m_{M}-\delta m_{N}$. Full process is $n M^{0} \rightarrow p M^{-} \rightarrow$ $p M^{0} e^{-} \bar{v}_{e}$, and the absence of these signals in space detectors makes it possible to conclude that $\delta m_{M}>\delta m_{N}$.

\section{Interaction of New Hadrons with Leptons}

To describe the interaction of new heavy hadrons with leptons we have to construct effective vertex of type $M_{1} M_{2} V$, where $M_{a}$ is $M^{0}$ or $M^{-}$and $V=Z, W$. From the quark content of $M$-mesons, $M=(Q, q)$, it follows that the vertex $M M Z$ is caused by interaction of $Z$-boson with both quarks, $Q$ and $q$, so we should take into consideration the interference of these sub-processes. In contrast, the vertex $M_{1} M_{2} W$ is caused by interaction of $W$-boson with standard quark $q$ only. So, the last vertex can be defined in form-factor approach (exclusive process) or in spectator approach (inclusive process). In Refs. [5,6], we described the decay $M^{-} \rightarrow M^{0} W^{-} \rightarrow M^{0} e^{-} \bar{v}_{e}$ where $M^{0}=(\bar{U} u)$ and $M^{-}=(\bar{U} d)$ in analogy with standard heavy-light meson decay. Using a known method of calculation (see review by R. Kowalski in [16]) in HQS approximation, we derived the width of decay in the form [5]:

$$
\Gamma\left(M^{-} \rightarrow M^{0} e^{-} \bar{v}_{e}\right) \approx \frac{G_{F}^{2} \delta m^{5}}{60 \pi^{3}}=\frac{g^{4} \delta m}{15 \times 2^{7} \pi^{3}}\left(\frac{\delta m}{M_{W}}\right)^{4},
$$

where $g$ is the constant of weak interaction and $\delta m=m^{-}-m^{0}$. In Ref. [6], the same formula was derived in the form-factor approach which will be used further. In this approach, the amplitude of decay at the quark level $A \sim G_{F} \bar{e} \gamma^{\mu}\left(1-\gamma_{5}\right) v \cdot \bar{u} \gamma_{\mu}\left(1-\gamma_{5}\right) d$, is transformed into the amplitude at the meson level $\left\langle M^{0}|A| M^{-}\right\rangle$with the help of equality:

$$
\left\langle M^{0}\left|\bar{u} \gamma_{\mu}\left(1-\gamma_{5}\right) d\right| M^{-}\right\rangle \approx f_{+}^{M}\left(q^{2}\right)\left(P_{M}^{-}+P_{M}^{0}\right)_{\mu},
$$

where $f_{+}^{M}\left(q^{2}\right)$ is form-factor as the function of momentum transfer, $P_{M}^{-}$and $P_{M}^{0}$ are the four-momentum of heavy meson in initial and final states. Using the expression (26), smallness of $q^{2}$ and $f_{+}^{M}\left(q^{2}\right) \approx$ $f_{+}^{M}(0)=1$, we have found the expression for width (25). Here, this result we use for determination of the effective coupling constant in the vertex $M M W$, which we represent in the following differential form:

$$
L^{e f f}(W M M)=i G_{W M} W^{\mu}\left(\bar{M}^{0} \partial_{\mu} M^{-}-\partial_{\mu} \bar{M}^{0} M^{-}\right) .
$$

Note, the Lagrangian in the differential form (27) generates the vertex which has the same structure in the momentum representation as vertex in form-factor approach (26). Using effective Lagrangian of interaction (27), we calculate $\Gamma\left(M^{-} \rightarrow M^{0} e^{-} \bar{v}_{e}\right)$ and found the value of effective constant $G_{W M}$ by matching the result with Formula (25). Calculation of width is fulfilled in the framework of the model of unstable particles with smeared mass $[17,18]$. For the case of the decay $1 \rightarrow 3$ the expression for width is described by convolution representation [17]:

$$
\Gamma\left(M^{-} \rightarrow M^{0} e^{-} \bar{v}_{e}\right)=\int_{q_{1}^{2}}^{q_{2}^{2}} \Gamma\left(M^{-} \rightarrow M^{0} W(q)\right) \frac{q \Gamma\left(W(q) \rightarrow e^{-} \bar{v}_{e}\right)}{\pi\left|P_{W}(q)\right|^{2}} d q^{2},
$$

where $W(q)$ is intermediate state of $W$ with smeared mass $m^{2}=q^{2}$ and $P_{W}(q)$ is propagator of $W$-boson, which at $q^{2} \ll M_{W}^{2}$ is $P_{W}(q) \approx-M_{W}^{2}$. The rest integrands in (28) are calculated in standard way:

$$
\Gamma\left(M^{-} \rightarrow M^{0} W(q)\right)=\frac{G_{W M^{2}}^{2} m_{-}^{3}}{16 \pi q^{2}} \bar{\lambda}^{3}\left(q^{2}, m_{0}^{2} ; m_{-}^{2}\right),
$$

where normalized kinematic function $\bar{\lambda}$ is defined as follows:

$$
\bar{\lambda}^{3}\left(q^{2}, m_{0}^{2} ; m_{-}^{2}\right)=\left(1-2 \frac{q^{2}+m_{0}^{2}}{m_{-}^{2}}+\frac{\left(m_{0}^{2}-q^{2}\right)^{2}}{m_{-}^{4}}\right)^{1 / 2} .
$$


Then, for the width of the decay $W(q) \rightarrow e^{-} \bar{v}_{e}$ we have:

$$
\Gamma\left(W(q) \rightarrow e^{-} \bar{\nu}_{e}\right)=\frac{g^{2} q}{48 \pi} \bar{\lambda}\left(o, m_{e}^{2} ; q^{2}\right)\left(1-\frac{m_{e}^{2}}{2 q^{2}}-\frac{m_{e}^{4}}{2 q^{4}}\right) .
$$

Further, taking into account relations $m_{-}=m_{0}+\delta m$ and $q^{2} \sim(\delta m)^{2} \ll m_{0}^{2}$, we represent Equation (28) in the form:

$$
\Gamma\left(M^{-} \rightarrow M^{0} e^{-} \bar{v}_{e}\right)=\frac{g^{2} G_{W M}^{2}}{96 \pi^{3} M_{W}^{4}} \int_{m_{e}^{2}}^{(\delta m)^{2}}\left((\delta m)^{2}-q^{2}\right)^{3 / 2} d q^{2} .
$$

In the approximation $m_{e}^{2} \ll(\delta m)^{2}$, the width is as follows:

$$
\Gamma\left(M^{-} \rightarrow M^{0} e^{-} \bar{v}_{e}\right) \approx \frac{g^{2} G_{W M}^{2}}{15 \cdot 2^{4} \pi^{3}} \frac{(\delta m)^{5}}{M_{W}^{4}} .
$$

Comparison of the expressions (25) and (33) leads to the relation $G_{W M}^{2}=g^{2} / 2^{3}$ or $G_{W M}=g / 2 \sqrt{2}$. It should be noted, the value of effective coupling constant $G_{W M}$ in Equation (27) is the same as fundamental constant in the interaction of $W$-boson with quark current $\bar{u} \gamma_{\mu}\left(1-\gamma_{5}\right) d$. This means that spectator approach at small momentum transfer is valid and corresponds to the structure of subprocess at the quark level.

The scattering of low-energy lepton on the hadronic DM is defined by one $t$-channel diagram with $W$-boson in the intermediate state. This process can be described with help of the effective Lagrangian (27) with constant coupling $G_{W M}=g / 2 \sqrt{2}$ and standard vertex of type $W l \bar{v}_{l}$, where $l=e, \mu, \tau$. From straightforward calculation it results the following form of the cross-section:

$$
\sigma\left(e^{-} M^{0} \rightarrow v_{e} M^{-}\right)=\frac{3 g^{4}}{2^{10} \pi M_{W}^{4}} s\left(1-\frac{M^{2}}{s}\right)^{2},
$$

where $\sqrt{s}$ is full energy in CMS. For non-relativistic DM, this expression can be represented as:

$$
\sigma\left(e^{-} M^{0} \rightarrow v_{e} M^{-}\right)=\frac{3 G_{F}^{2}}{8 \pi}\left(E_{e}+W_{k}\right)^{2},
$$

where $E_{e}$ is the electron energy and $W_{k}=M v^{2} / 2$ is kinetic energy of DM particle. The full process of electron scattering on $M^{0}$ is: $e^{-} M^{0} \rightarrow v_{e} M^{-} \rightarrow v_{e} M^{0} e^{-} \bar{v}_{e}$, that is the process with neutrino-antineutrino pair in the final state.

\section{Conclusions}

The main purpose of the DM investigation is the description of the processes of its interaction with ordinary matter and predictions of possible signals. In order to describe signals from the hadronic $\mathrm{DM}$, we have developed and analyzed in details the effective model of new hadrons interaction with nucleons and leptons. The model of DM-nucleon low-energy interaction is based on meson-exchange approach and realized in gauge scheme with $U(1) \cdot S U(3)$ symmetry. In the framework of this model, we derived analytical expressions for cross-sections of collisions of nucleons and new hadrons. These expressions describe both elastic and inelastic processes of scattering of nucleons on new heavy hadrons. They can be used for the analysis and investigations of the DM interactions with interstellar gas in the Galaxy. The most important signals of the processes with participation of DM are shortly considered.

To describe the interaction of hadronic DM with leptons we have constructed the effective vertexex of type WMM. From the quark content of $M$-meson, it follows that the vertex $Z M M$ resulted from the interaction of $Z$-boson with both quarks, $Q$ and $q$, while the vertex $W M M$ is caused by the interaction of $W$-boson with standard light quark $q$ only. In the previous work, the decay 
process $\mathrm{M}^{-} \rightarrow \mathrm{M}^{0} \mathrm{~W}^{-} \rightarrow \mathrm{M}^{0} e^{-} \bar{v}_{e}$ was analyzed in formfactor approach. Here, using this result, we constructed an effective Lagrangian of WMM-interaction and defined the effective coupling constant. This Lagrangian was used for calculation of the cross-section of lepton scattering on new heavy hadrons. It is shown that there appears some typical signature of this processes which can be registered.

Author Contributions: The authors contributed equally to the work. All authors have read and agreed to the published version of the manuscript.

Funding: This research was funded by grant of the Russian Science Foundation, grant number 18-12-00213.

Acknowledgments: Authors are sincerely grateful to M. Khlopov for fruitful cooperation in scientific research.

Conflicts of Interest: The authors declare no conflict of interest.

\section{References}

1. Aprile, E.; Aalbers, J.; Agostini, F.; Alfonsi, M.; Amaro, F.D.; Anthony, M.; Arneodo, F.; Barrow, P.; Baudis, L.; Bauermeister, B.; et al. First Dark Matter Search Results from the XENON1 Experiment. Phys. Rev. Lett. 2017, 119, 181301. [CrossRef] [PubMed]

2. McGuire, P.C.; Steinhardt, P.G. Cracking Open the Window for Strongly Interacting Massive Particles as the Halo Dark Matter. arXiv 2001, arXiv:astro-ph/0105567.

3. Huo, R.; Matsumoto, S.; Tsai, J.L.S.; Yanagida, T.T. A scenario of heavy but visible baryonic dark matter. JHEP 2016, 1609, 162-166. [CrossRef]

4. Luca, V.; Mitridate, A.; Redi, M.; Smirnov, J.; Strumia, A. Colored Dark Matter. Phys. Rev. D 2018, 97, 115024. [CrossRef]

5. Beylin, V.; Kuksa, V. Dark Matter in the Standard Model Extention with Singlet Quark. Adv. High Energy Phys. 2018, 2018, 8670954. [CrossRef]

6. Beylin, V.; Kuksa, V. Possibility of hadronic dark matter. Int. J. Mod. Phys. D 2018, 28, 1941001. [CrossRef]

7. Beylin, V.; Khlopov, M.Y.; Kuksa, V.; Volchanskiy, N. Hadronic and Hadron-Like Physics of Dark Matter. Symmetry 2019, 11, 587. [CrossRef]

8. Bazhutov, Y.N.; Vereshkov, G.M.; Kuksa, V.I. Experimental and Theoretical Premises of New Stable Hadron Existence. Int. J. Mod. Phys. A 2017, 2, 1759188. [CrossRef]

9. Khlopov, M.Y. Physics of dark matter im the light of dark atoms. Mod. Phys. Lett. A 2011, 26, $2823-2839$. [CrossRef]

10. Khlopov, M.Y. Introduction to the special issue on indirect dark matter searches. Int. J. Mod. Phys. A 2014, 29, 1443002. [CrossRef]

11. Cudell, J.R.; Khlopov, M. Dark atoms with nuclear shell: A status review. Int. J. Mod. Phys. D 2015, 24, 1545007. [CrossRef]

12. Belotsky, K.M.; Fargion, D.; Khlopov, M.Y.; Konoplich, R.V.; Ryskin, M.G.; Shibaev, K.I. Heavy hadrons of 4th family hidden in our Universe and close to detection. Grav. Cosmet. Suppl. 2005, 11, 3-15.

13. Walecka, J.D. A Theory of Condensed Matter. Ann. Phys. 1974, 83, 491-529. [CrossRef]

14. Serot, B.D. A relativistic nuclear field theory with $\pi$ and $\rho$ mesons. Phys. Lett. B 1979, 86, 146-150. [CrossRef]

15. Vereshkov, G.M.; Kuksa, V.I. U(1)SU(3)-gauge model of baryon-meson interactions. Yad. Fiz. 1991, 54, 1700-1704.

16. Tanabashi, M.; Hagiwara, K.; Hikasa, K.; Nakamura, K.; Sumino, Y.; Takahashi, F.; Tanaka, J.; Agashe, K.; Aielli, G.; Amsler, C.; et al. The Review of Particle Physics. Phys. Rev. D 2018, 98, 1. [CrossRef]

17. Kuksa, V.I. The convolution formula for a decay rate. Phys. Lett. B 2006, 633, 545-549. [CrossRef]

18. Kuksa, V.I.; Volchanskiy, N.I. Factorization in the model of unstable particles with continuous masses. Cent. Eur. J. Phys. 2013, 11, 182-194. [CrossRef]

(C) 2020 by the authors. Licensee MDPI, Basel, Switzerland. This article is an open access article distributed under the terms and conditions of the Creative Commons Attribution (CC BY) license (http:/ / creativecommons.org/licenses/by/4.0/). 